\title{
Effects of mate tea consumption on muscle strength and oxidative stress markers after eccentric exercise
}

\author{
Vilma Pereira Panza ${ }^{1}$, Fernando Diefenthaeler ${ }^{2}$, Aline Cavalheiro Tamborindeguy ${ }^{2}$, \\ Carolina de Quadros Camargo ${ }^{1,3}$, Bruno Monteiro de Moura ${ }^{2}$, Henver Simionato Brunetta ${ }^{3}$, \\ Raphael Luiz Sakugawa ${ }^{2}$, Marina Vieira de Oliveira ${ }^{4}$, Emiliana de Oliveira Puel ${ }^{4}$, Everson Araújo Nunes ${ }^{1,3}$ \\ and Edson Luiz da Silva ${ }^{1,4 *}$ \\ ${ }^{1}$ Nutrition Post-Graduate Program, Federal University of Santa Catarina, Florianopolis, Santa Catarina, ZC 88040-970, Brazil \\ ${ }^{2}$ Biomechanics Laboratory, Department of Physical Education, Federal University of Santa Catarina, Florianopolis, Santa \\ Catarina, ZC 88040-970, Brazil \\ ${ }^{3}$ Laboratory of Investigation in Chronic Diseases, Department of Physiological Sciences, Federal University of Santa Catarina, \\ Florianopolis, Santa Catarina, ZC 88040-970, Brazil \\ ${ }^{4}$ Laboratory of Lipids, Antioxidants, and Atherosclerosis, Department of Clinical Analysis, Federal University of Santa \\ Catarina, Florianopolis, Santa Catarina, ZC 88040-970, Brazil
}

(Submitted 5 August 2015 - Final revision received 15 December 2015 - Accepted 24 January 2016 - First published online 26 February 2016)

\section{Abstract}

Dietary phytochemical supplementation may improve muscle recovery from exercise. In this study, we investigated the effect of mate tea (MT) consumption - a phenol-rich beverage - on muscle strength and oxidative stress biomarkers after eccentric exercise. In a randomised, crossover design, twelve men were assigned to drink either MT or water (control; CON) for $11 \mathrm{~d}$. On the 8th day, subjects performed three sets of twenty maximal eccentric elbow flexion exercises. Maximal isometric elbow flexion force was measured before and at $0,24,48$ and $72 \mathrm{~h}$ after exercise. Blood samples were obtained before and at 24, 48 and $72 \mathrm{~h}$ after exercise and analysed for total phenolics, GSH, GSSG, GSH:GSSG ratio and lipid hydroperoxides (LOOH). After eccentric exercise, muscle strength was significantly reduced over time, regardless of treatments. However, MT improved the rate of strength recovery by $8.6 \%$ on the 1 st day after exercise $(P<0.05)$. Plasma concentration of total phenolic compounds was higher in MT than in CON at all time points $(P<0.05)$ but decreased significantly at $72 \mathrm{~h}$ after exercise in both trials $(P<0.05)$. Blood levels of GSH were significantly decreased at 48 and $72 \mathrm{~h}$ after exercise in CON $(P<0.05)$ but did not change over time in MT. No significant changes were observed for GSSG, GSH:GSSG ratio and LOOH levels. MT intake did not influence muscle strength at all time points assessed but hastened the strength recovery over $24 \mathrm{~h}$ after exercise. MT also favoured the concentration of blood antioxidant compounds.

Key words: Ilex paraguariensis: Mate tea: Phenolic compounds: Eccentric exercise: Muscle damage: Isometric strength: Oxidative stress

During an eccentric muscle action - such as while lowering a dumbbell - the muscle is being forcibly lengthened while generating force ${ }^{(1)}$. It is well established that exercises that involve unaccustomed and/or repeated eccentric actions may result in muscle damage and immediate decline in muscle strength $^{(1-4)}$. The complete restoration of muscle function can take from a few to several days after exercise, depending on the extent of the damage ${ }^{(2-4)}$. It has been assumed that the delayed recovery of muscle strength may be in part associated with further damages elicited by reactive molecules released within the injured muscle ${ }^{(5-8)}$.

There is growing evidence that supplementation with foods rich in phytochemicals that have antioxidant and/or anti-inflammatory properties may improve the recovery from eccentric exercise-induced muscle damage (EEIMD) ${ }^{(9-12)}$. Having several choices of dietary strategies for strength recovery can be quite advantageous for sportspeople. In this sense, the consumption of mate tea (MT), a beverage made from an infusion of roasted leaves of yerba mate (Ilex paraguariensis), might be an interesting option. Yerba mate is a plant originally from South America that represents a good source of phytochemicals with antioxidant and anti-inflammatory properties, such as phenolic compounds and saponins ${ }^{(13)}$. The consumption of MT or green yerba mate infusion leads to an increase in antioxidant defence in plasma and in immune cells in humans ${ }^{(14-16)}$. Acute or regular consumption of yerba mate-based beverages such as

Abbreviations: CON, control; EEIMD, eccentric exercise-induced muscle damage; LOOH, lipid hydroperoxides; MT, mate tea; PRE, pre-exercise.

* Corresponding author: E. L. d. Silva, email edson.silva@ufsc.br 
MT and green yerba mate infusion has shown improvements related to clinical disorders such as dyslipidaemia, diabetes and obesity $^{(13-16)}$. On the other hand, information about the benefits of yerba mate regarding exercise is scarce. It was recently demonstrated that acute supplementation with ground yerba mate leaves improved fat oxidation during submaximal aerobic exercises in healthy subjects ${ }^{(17)}$. In an animal study, treatment with yerba mate extract over a 4-week swimming training attenuated lipid peroxidation, as well as increased superoxide dismutase enzyme activity in muscles and the liver ${ }^{(18)}$. However, the effects of MT intake on the recovery of muscle function after damaging exercise are still unknown.

Therefore, the primary purpose of this study was to investigate whether MT supplementation influences the recovery of muscle strength after a bout of eccentric exercise in physically active subjects. We also assessed the effects of MT on blood oxidative stress biomarkers following exercise.

\section{Methods}

\section{Subjects}

In all, twelve healthy and physically active male students (25.1 (sD 3.6) years, 75.1 (sD 9.8) kg, 175.8 (sD 5.8) cm) were recruited from the Department of Physical Education of a local University. The volunteers were selected according to the following criteria: non-athletes; not having participated in resistance training programme in the previous 3 months; not been involved in any structured endurance training programme; non-smokers; free of any disease, infection or inflammatory processes; not having a history of upper limb injury; and not using any medication, herbals, ergogenic aids or antioxidant supplements. All the experimental procedures were explained to the subjects before obtaining a signed written agreement. The study was approved by the Ethics Committee on Human Research of the Federal University of Santa Catarina. The trial was registered as RBR-5pj5bb.

\section{Experimental design}

This study was a randomised, controlled, cross-over trial. The experimental design is displayed in Fig. 1. The trial comprised two 11-d treatment periods (P1 and P2) with a 17-d washout period between each treatment (Fig. 1). Participants were randomly assigned to initiate P1, where they drank $200 \mathrm{ml}$ of either MT or water (control, CON). The beverages were consumed three times per day (morning, afternoon and night). On the morning of the 8th day, subjects were instructed to drink one of the three daily doses of MT or CON $1 \mathrm{~h}$ before exercise tests. After this, all the subjects performed a damaging eccentric exercise protocol with one arm, which was chosen randomly. The maximal isometric force (i.e. isometric strength) of elbow flexor muscles was measured before (PRE) and at 0, 24, 48 and $72 \mathrm{~h}$ after exercise. Blood samples were obtained at PRE and at 24,48 and $72 \mathrm{~h}$ after exercise, before the isometric strength test. During the second test period (P2), exercise tests were performed with the contralateral arm. All the participants were instructed to eat a standardised breakfast composed of skimmed milk, sugar, white bread, honey and bananas $2 \mathrm{~h}$ before the exercise tests. All the subjects maintained their usual physical activities, except in the $24 \mathrm{~h}$ before the eccentric exercise bout, during which they were asked to refrain from intense activities using the upper limbs.

\section{Mate tea preparation and intake}

Participants received a completely sealed 50-g package of lyophilised instant MT (Leão Alimentos e Bebidas ${ }^{\circledR}$ ). Instructions for preparing and drinking MT were given verbally and in writing. MT was prepared by dissolving $1 \mathrm{~g}$ (one teaspoon) of instant MT in $200 \mathrm{ml}$ of cold water $(5 \mathrm{mg} / \mathrm{ml})$, according to the manufacturer's instructions. No sugar, sweetener or fruits were allowed to be added to the beverage.

\section{Determination of total phenolics of mate tea}

The total phenol content of MT was measured according to the modified Folin-Ciocalteu method as described by Singleton et $a{ }^{(19)}$. In brief, $300 \mu \mathrm{l}$ of MT was added to $1 \mathrm{ml}$ of $95 \%$ ethanol, $5 \mathrm{ml}$ of distilled water and $0.5 \mathrm{ml}$ of $50 \%$ Folin-Ciocalteau reagent. After $5 \mathrm{~min}, 1 \mathrm{ml}$ of $5 \%$ sodium bicarbonate was added. The mixture was left at room temperature in the absence of light for $1 \mathrm{~h}$. The absorbance of the coloured product was measured at $765 \mathrm{~nm}$, and 3-caffeoylquinic acid was used as the standard. The average inter-assay CV for triplicate preparations was $7 \cdot 3 \%$.

The phenolic acids of MT were determined by HPLC (Shimadzu LC-10) as described by Strassmann et al. ${ }^{(20)}$.

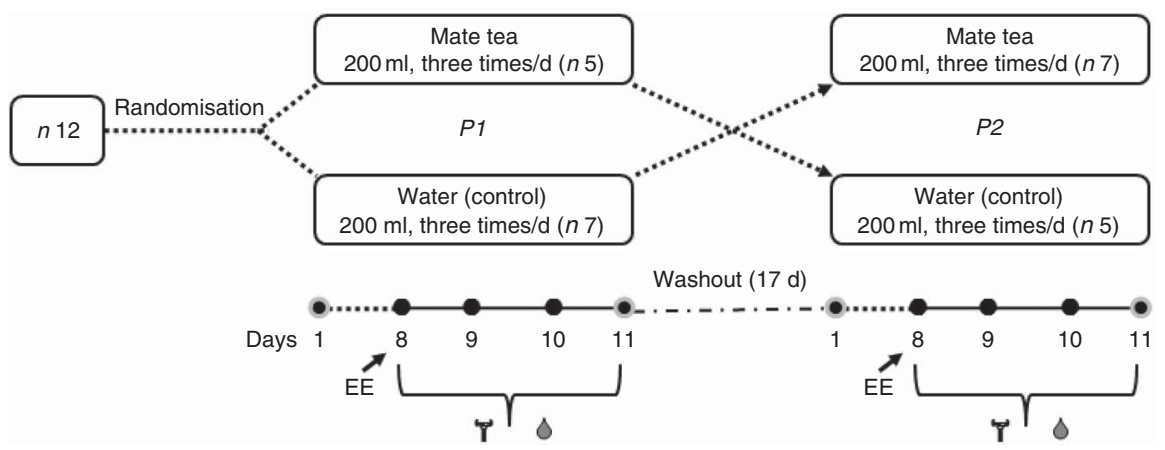

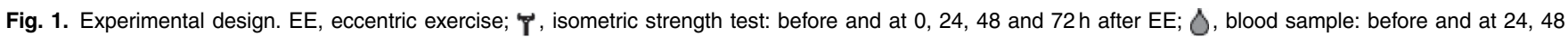
and $72 \mathrm{~h}$ after EE. 
The instant MT was prepared and filtered through a $0.45-\mu \mathrm{m}$ micropore membrane, and aliquots were injected into a $\mathrm{C} 18$ reverse-phase column $(4.6 \times 250 \mathrm{~mm}, 5-\mu \mathrm{m}$; Shim-pack $)$ with a Shim-pack C18 guard column $(4.0 \mathrm{~mm} \times 10 \mathrm{~mm}, 5 \mu \mathrm{m})$. An isocratic mobile phase consisting of $n$-butanol-acetic acid-water $(97 \cdot 0: 0 \cdot 28: 2 \cdot 72, \mathrm{v} / \mathrm{v} / \mathrm{v})$ was used at a flow rate of $0 \cdot 8 \mathrm{ml} / \mathrm{min}$. The assay was monitored at $325 \mathrm{~nm}$. The concentrations of phenolic acids were quantified by 5-point calibration curves of standards. Gallic, caffeic, 3-caffeoylquinic and 4,5-dicaffeoylquinic acids were used as standards. The final concentration of phenolic compounds was determined by averaging the results of three consecutive injections.

\section{Determination of total saponins in mate tea}

The content of total saponins in MT was measured by a spectrophotometric method after acid hydrolysis of the MT saponins and extraction of sapogenins, as described by Gnoatto et al. ${ }^{(21)}$, and reaction with vanillin and perchloric acid, according to Fan $\& \mathrm{He}^{(22)}$. Ursolic acid, the major triterpenic nucleus of saponins present in I. paraguariensis ${ }^{(21)}$, was used as a standard, and the results are expressed as milligram equivalents of ursolic acid per millilitre. The inter-assay CV of total saponins of the three MT preparations was $8 \cdot 1 \%$.

\section{Eccentric exercise}

The eccentric exercise protocol was performed on an isokinetic dynamometer (Biodex System-4Pro ${ }^{\circledR}$; Biodex Medical Inc.). Subjects performed three sets of twenty repetitions of unilateral, maximal, isokinetic eccentric actions of the elbow flexor muscles at an angular velocity of $45^{\circ}$ s, with 2 -min rest between sets. The range of motion was from 50 to $170^{\circ}$ of full elbow flexion. Subjects were seated with their elbow on a Scott Bench (Troya), and the axis of rotation was aligned with the lateral epicondyle of the humerus. Each repetition lasted $3 \mathrm{~s}$. During the exercise, participants were verbally encouraged to put in maximal effort. Total work was recorded for each set.

\section{Isometric strength test}

To evaluate the maximal force generation of the elbow flexors, participants performed three maximum voluntary isometric muscle actions at $90^{\circ}$, each lasting $3 \mathrm{~s}$, with 2 min of rest between sets. Strength was reported as the highest peak torque recorded at each trial. Subjects were verbally encouraged throughout the test. Before the tests, subjects warmed up by actively flexing and extending the elbow joint (10 repetitions) at $120 \%$ s. All the volunteers participated in familiarisation tests on the isokinetic dynamometer before the beginning of the study. Isometric strength was expressed as a percentage of pre-exercise levels. The rate of isometric strength recovery was calculated by subtracting the value of the strength at 24,48 or $72 \mathrm{~h}$ after exercise from the value of the strength immediately after eccentric exercise $(0 \mathrm{~h})$.

\section{Blood sampling}

Blood samples were collected between 07.00 and 08.00 hours, before any exercise test and after $15 \mathrm{~min}$ of rest in a sitting position. The exercise test protocols started at 08.00 hours. Samples were obtained from the contralateral arm that was being tested. The median antecubital vein was punctured using a hypodermic needle $(25 \times 7 \mathrm{~mm})$ and blood was collected into Vacutainer ${ }^{\circledR}$ tubes containing either EDTA, heparin or without any anticoagulants. Plasma and serum samples were obtained by centrifugation $\left(1000 \mathrm{~g}\right.$ for $10 \mathrm{~min}$, at $4^{\circ} \mathrm{C}$ ). For the analysis of glutathione status, EDTA whole blood $(1 \mathrm{ml})$ was transferred to an Eppendorf tube containing $100 \mu \mathrm{l}$ of $310 \mathrm{~mm}-\mathrm{N}$-ethylmaleimide (NEM310; Sigma-Aldrich), vortex-mixed and frozen at $-80^{\circ}$ C for later analysis.

\section{Dietary intake}

Participants maintained their usual dietary pattern throughout the study, except for the restriction (more than three times a week) of beverages with known antioxidant properties, such as black tea, green tea, wine and fruit juices; 3 -d food records ( 1 weekend day and 2 non-consecutive days during the week) were collected during each of the two experimental periods of the study (i.e. P1 and P2). Nutrient intakes were analysed for energy, macronutrients, vitamins (A, C and E) and minerals ( $\mathrm{Zn}, \mathrm{Cu}, \mathrm{Mn}$ and Se) intakes (Avanutri 4.0).

\section{Total phenolics in plasma}

Total phenolic compounds in plasma were measured by the Folin-Ciocalteau colorimetric method, according to the methodology described by Serafini et al. ${ }^{(23)}$. In brief, $500 \mu$ l of duplicate plasma samples were acidified and, after extraction of complexed phenols with alcoholic sodium hydroxide, proteins were precipitated using $0.75 \mathrm{~m}$-metaphosphoric acid and re-extracted with a mixture of acetone-water (1:1). Next, aliquots $(50 \mu \mathrm{l})$ of the samples were added to $0.5 \mathrm{ml}$ of Folin-Ciocalteau reagent (50\%). After $5 \mathrm{~min}, 1 \mathrm{ml}$ of $5 \%$ sodium bicarbonate was added. The mixture was left at room temperature in the dark for $1 \mathrm{~h}$. The absorbance of the coloured product was measured at $765 \mathrm{~nm}$, and chlorogenic acid was used as a standard. The average interassay CV for duplicate preparations was $6.8 \%$.

\section{Glutathione status}

GSH was determined in the whole blood by HPLC, according to the procedures described by Giustarini et $a l{ }^{(24)}$. Aliquots of the EDTA whole blood treated with NEM310 (see the Blood sampling section) were de-proteinised by the addition of $15 \%$ TCA and then centrifuged at $14000 \boldsymbol{g}$ at room temperature for 2 min. The supernatant was analysed by HPLC using a C18 column (TSK-gel ODS-80Ts; Tosoh, $4.6 \mathrm{~mm} \times 150 \mathrm{~mm} \times 5 \mu \mathrm{m}$ ). The mobile phase was composed of $0.25 \%$ acetic acid and acetonitrile $(94: 6, \mathrm{v} / \mathrm{v})$ at a flow rate of $1.25 \mathrm{ml} / \mathrm{min}$. Signals were recorded at $265 \mathrm{~nm}$ with $400 \mathrm{~nm}$ as the reference. GSH content was quantified through calibration GSH curve standards of 5 points and linear regression analysis.

GSSG was determined in the whole blood by spectrophotometry, according to Giustarini et $a l^{(24)}$. Aliquots of the EDTA whole blood treated with NEM310 were de-proteinised and centrifuged as described above. Next, the supernatant was 
extracted with three volumes of dichloromethane. The mixture was vortexed for $5 \mathrm{~min}$ and centrifuged at $14000 \mathrm{~g}$ at room temperature for $30 \mathrm{~s}$. The supernatant $(20 \mu \mathrm{l}), 925 \mu \mathrm{l}$ of $200 \mathrm{~mm}-\mathrm{PBS}$, $5 \mu \mathrm{l}$ of $20 \mathrm{~mm} 5,5^{\prime}$-dithiobis-2-nitrobenzoic acid, $20 \mu \mathrm{l}$ of $7.5 \%$ TCA with $75.0 \mathrm{mg} / \mathrm{ml}$ EDTA and $20 \mu \mathrm{l}$ of $4.8 \mathrm{~mm}$ NADPH were transferred to an assay tube and the absorbance was recorded (Spectrophotometer UV-1800; Shimadzu) at $412 \mathrm{~nm}$ for $1 \mathrm{~min}$ (blank). Subsequently, $20 \mu \mathrm{l}$ of $20 \mathrm{IU} / \mathrm{ml}$ glutathione reductase was added to the mixture, and the enzyme kinetic reaction was monitored at $412 \mathrm{~nm}$ for $1 \mathrm{~min}$. Next, $10 \mu \mathrm{m}-\mathrm{GSSG}$ was added to the tube, and the absorbance was recorded at $412 \mathrm{~nm}$ for 1 additional minute. GSSG concentration was calculated by the formula (GSSGb = $\mathrm{S} \times(\mathrm{GSSGC}) / \mathrm{St} \times 49.5 \times 2)$, where $\mathrm{S}=\Delta$ sample $-\Delta$ blank; $\mathrm{St}=$ $(\Delta$ sample $+\Delta$ GSSG $)-\Delta$ sample $; 49 \cdot 5=$ dilution factor of sample in the cuvette; and $2=$ dilution factor due to sample acidification. The results of both GSH and GSSG were normalised per gram of $\mathrm{Hb}$, which was measured by colorimetric end point assay. In brief, an aliquot of whole blood was mixed with Drabkin reagent, and the absorbance was read at $540 \mathrm{~nm}$ after $5 \mathrm{~min}$ at room temperature.

\section{Lipid hydroperoxides}

Lipid hydroperoxides (LOOH) were determined in heparinplasma using ferrous oxidation-xylenol orange (FOX2), as described by Nourooz-Zadeh et al. ${ }^{(25)}$. The method is based on the fast oxidation of $\mathrm{Fe}^{+2}$ to $\mathrm{Fe}^{+3}$ in acid medium mediated by lipid peroxides. In the presence of xylenol orange, $\mathrm{Fe}^{+3}$ forms a complex $\left(\mathrm{Fe}^{+3}\right.$-xylenol orange), which is measured spectrophotometrically at $560 \mathrm{~nm}$. FOX2 reagent, containing $250 \mathrm{~mm}-\mathrm{H}_{2} \mathrm{SO}_{4}, 4.4 \mathrm{~mm}$-butylated hydroxytoluene, $1 \mathrm{~mm}$-xylenol orange, and $2.5 \mathrm{~mm}$-iron ammonium sulphate in methanol, was added to aliquots of plasma, in duplicate. In addition, $10 \mu \mathrm{l}$ of 10 mm-triphenylphosphine in methanol was added to two additional microtubes with plasma to reduce $\mathrm{LOOH}$ before the addition of FOX2 reagent, thereby generating a blank sample. Subsequently, the mixtures were kept at room temperature for $30 \mathrm{~min}$, the tubes were centrifuged $(1000 \mathrm{~g}, 5 \mathrm{~min})$ and the absorbance was measured (Shimadzu). A standard hydrogen peroxide curve was used to quantify $\mathrm{LOOH}$ and the results are expressed in $\mu \mathrm{mol} / \mathrm{l}$ equivalent of hydrogen peroxide. The inter-assay $\mathrm{CV}$ calculated by the measurement of hydrogen peroxide on different days was $10.2 \%$. The average $\mathrm{CV}$ percentage for duplicate variation was $6.7 \%$.

\section{Sample size and statistical analysis}

To our knowledge, this was the first investigation on the effects of MT supplementation on the recovery of muscle strength after EEIMD. Thus, there is no information available about the sample size necessary to detect significant differences $(P<0 \cdot 05$; power of $80 \%$ ) between the MT and CON trials for muscle strength (the primary outcome). Previous, similar, cross-over studies have reported improvement in strength recovery in samples of ten to sixteen subjects ${ }^{(8,9,12)}$. Therefore, we decided to conduct this cross-over study with a sample size of twelve subjects (assuming 20\% loss). The Shapiro-Wilk test was applied to determine the normality of the data. When necessary, logarithmic transformation of data was used. Data that passed the normality check were analysed using a two-way repeatedmeasures ANOVA. Tukey's post hoc test was used for time, treatment and treatment $\times$ time analysis data. The Student's $t$ test was conducted to assess interactions between period and treatment (order effect). For data that did not pass the normality test, Friedman's test with Tukey's post hoc test was used for time analysis, and Wilcoxon's signed-rank test was performed to compare treatments at specific time points. Cohen's $d$ statistics $^{(26)}$, using the means and standard deviations ${ }^{(27)}$, were applied to assess treatment effect size for data that passed the normality test. Effect sizes were assumed as trivial $(<0 \cdot 20)$, small (between 0.20 and 0.49 ), medium (between 0.50 and 0.79 ) or large $(>0 \cdot 80)^{(26)}$. For data that did not pass the normality test, effect sizes were examined using Cliff's $\delta$ statistics. The amount of effect sizes was interpreted as trivial $(<0 \cdot 147)$, small (between 0.147 and 0.33 ), medium (between 0.33 and 0.474 ) or strong $(>0 \cdot 474)^{(28)}$. Retrospective power $(1-\beta)$ calculation $^{(29)}$ of effect size for the primary outcome with $n 12$ was carried out using G*Power $^{\circledR}$ (http://www.gpower.hhu.de/en.html), and the result indicated a power of 0.974 . All comparisons were analysed using SigmaPlot 12.0 (Systat Software Inc.). Statistical significance was set at $P \leq 0 \cdot 05$. Data are given as mean values and standard deviations or medians and interquartile ranges (25-75\%). Cohen's $d$ effect size was calculated using an effect size calculator (http://www.cem.org/effect-size-calculator). Cliff's $\delta$ effect size was computed using Cliff's Delta Calculator software ${ }^{(30)}$.

\section{Results}

All twelve participants who were recruited completed both intervention arms and were included in the data analysis. The assessment of the participants' 3-d dietary records showed that there were no significant differences between the MT and CON trials for energy and the analysed nutrients intakes (Table 1).

\section{Quantitative analysis of mate tea}

Total phenols, phenolic compounds and saponin contents in MT are given in Table 2. Considering that the participants had a daily intake of $3 \mathrm{~g}$ lyophilised instant mate, the mean intakes of MT phenolic compounds and saponins were 890 and $142 \mathrm{mg} / \mathrm{d}$, respectively. Chlorogenic acids were the major phenolic compounds found in MT followed by gallic acid and caffeic acid.

\section{Muscle strength}

There was no significant difference in the eccentric work performed between treatments (MT, 2454 (SD 149) J $v$. CON 2498 (sD 495) J; $d=-0 \cdot 12)$. Immediately after eccentric exercise ( $0 \mathrm{~h})$, isometric strength was significantly below pre-exercise levels $(P<0 \cdot 001)$, with no difference between MT and CON (MT 62.3 (SD 10.4) \% $v$. CON 63.2 (SD 11.8) \% of PRE; $d=-0.08$ ) (Fig. 2). Strength remained significantly reduced from 24 to $72 \mathrm{~h}$ after exercise, regardless of the treatment used. Nevertheless, there was a medium effect size $(d=0.53)$ for isometric strength at $24 \mathrm{~h}$ after exercise. In addition, from 0 to $24 \mathrm{~h}$ after exercise, the 
Table 1. Dietary intake* in the mate tea (MT) and control trials (Mean values and standard deviations)

\begin{tabular}{|c|c|c|c|c|}
\hline Variables & MT & SD & Control & SD \\
\hline Energy (kJ) & 9887.6 & $2340 \cdot 1$ & 9520.7 & $2737 \cdot 2$ \\
\hline Energy (kcal) & $2363 \cdot 2$ & $559 \cdot 3$ & 2275.5 & 654.2 \\
\hline Carbohydrate (g) & $306 \cdot 7$ & $101 \cdot 2$ & $302 \cdot 6$ & $112 \cdot 5$ \\
\hline Protein (g) & $123 \cdot 2$ & 24.5 & $121 \cdot 1$ & $30 \cdot 1$ \\
\hline Lipid (g) & $76 \cdot 5$ & $12 \cdot 2$ & $72 \cdot 4$ & $10 \cdot 8$ \\
\hline Vitamin E (mg) & $15 \cdot 7$ & $7 \cdot 3$ & $14 \cdot 8$ & $7 \cdot 8$ \\
\hline Vitamin C (mg) & $106 \cdot 2$ & $74 \cdot 0$ & $103 \cdot 3$ & 75.5 \\
\hline Vitamin A $(\mu \mathrm{g}) \dagger$ & $425 \cdot 8$ & 235.4 & $419 \cdot 1$ & $225 \cdot 0$ \\
\hline $\mathrm{Zn}$ (mg) & $15 \cdot 3$ & 3.5 & $14 \cdot 3$ & $3 \cdot 0$ \\
\hline $\mathrm{Cu}(\mu \mathrm{g})$ & 1065.5 & 282.9 & 1048.5 & $281 \cdot 0$ \\
\hline $\mathrm{Mn}(\mathrm{mg})$ & 2.5 & 0.6 & $2 \cdot 4$ & 0.4 \\
\hline $\mathrm{Se}(\mu \mathrm{g})$ & $122 \cdot 3$ & 33.4 & $120 \cdot 8$ & 41.4 \\
\hline
\end{tabular}

There was no statistical difference $(P>0.05)$ for any of the variables.

* Averages from 3-d food records.

$\dagger$ As retinol activity equivalents.

Table 2. Total phenol, phenolic compounds and saponin contents present in mate tea (MT)

(Mean values and standard deviations)

\begin{tabular}{lrr}
\hline MT compounds & $\mu \mathrm{g} / \mathrm{ml}^{*}$ & $\mathrm{SD}$ \\
\hline Total phenols & $1482 \cdot 3$ & 41.5 \\
5-Caffeoylquinic acid & $112 \cdot 7$ & $7 \cdot 6$ \\
3,5-Dicaffeoylquinic acid & $106 \cdot 3$ & $5 \cdot 8$ \\
4-Caffeoylquinic acid & $100 \cdot 5$ & $5 \cdot 5$ \\
3,4-Caffeoylquinic acid & 67.7 & $4 \cdot 2$ \\
3-Caffeoylquinic acid & 63.8 & $5 \cdot 1$ \\
4,5-Caffeoylquinic acid & 4.5 & 3.7 \\
Gallic acid & $16 \cdot 2$ & 1.4 \\
Caffeic acid & $2 \cdot 3$ & 0.01 \\
Total saponins & $236 \cdot 3$ & 9.6 \\
\hline
\end{tabular}

* Mean of triplicate measurements.

pattern of the recovery of strength was distinct between trials. At $24 \mathrm{~h}$, strength was significantly greater than that at $0 \mathrm{~h}$ only in the MT trial (Fig. 2; $P=0.008$ ). There were no order effects for either isometric strength values (data no shown).

In order to stress the evidences above - that MT may improve the isometric strength at $24 \mathrm{~h}$ after eccentric exercise - we re-analysed the data, taking into account the rate of muscle strength recovery. On the basis of the results, the rate of strength recovery during the $0-24-\mathrm{h}$ interval was higher in MT than in CON (15.3 (SD 9.2) \% and 6.7 (SD 6.1)\%, respectively; $P=0 \cdot 009)$ (Fig. 3), with a large effect size $(d=1 \cdot 11)$. The recovery rates at the $0-48$ - $\mathrm{h}$ and $0-72$-h intervals were not different between treatments $(P=0.277$ and 0.250 , respectively), with small effect sizes $(d=0.31$ and 0.41 , respectively).

\section{Biochemical variables}

A significant time $(P=0.011)$ and treatment $(P=0.008)$ effect occurred for the plasma concentration of total phenolic compounds (Fig. 4). In both treatments, total phenolics were unaffected from PRE to $48 \mathrm{~h}$ after exercise, and then decreased significantly from 48 to $72 \mathrm{~h}$ after exercise (MT, $P=0.040$ and

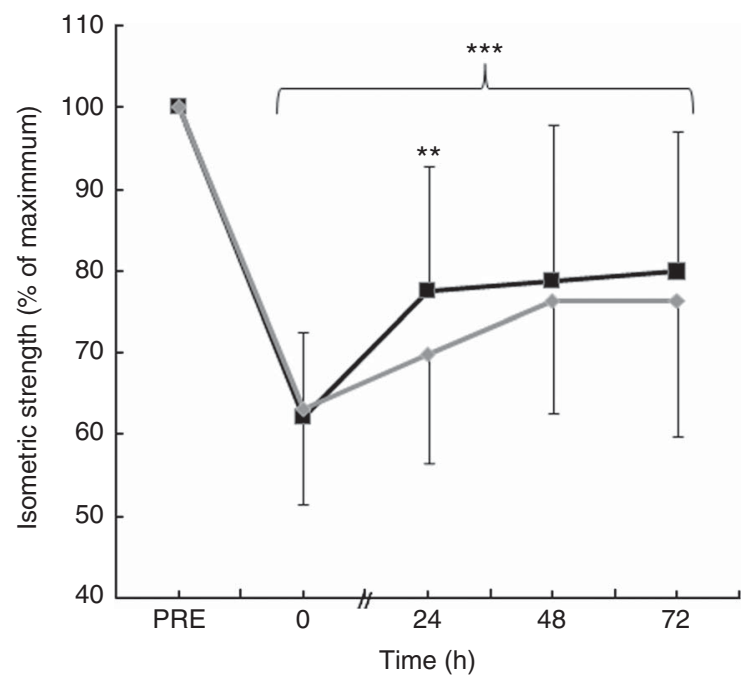

Fig. 2. Isometric elbow flexion strength (expressed as a percentage of preexercise levels) in the mate tea ( $\mathrm{MT},--$ ) and control (CON, - - ) trials. Treatments were consumed at a dosage of $200 \mathrm{ml}$, three times a day for $11 \mathrm{~d}$. Values are means and standard deviations. Two-way repeated-measures ANOVA and Tukey's post hoc test. Significant time effect: ${ }^{* *} P<0.001$. Significantly different from $0 \mathrm{~h}$ after exercise: ${ }^{\star *} P<0.01$.

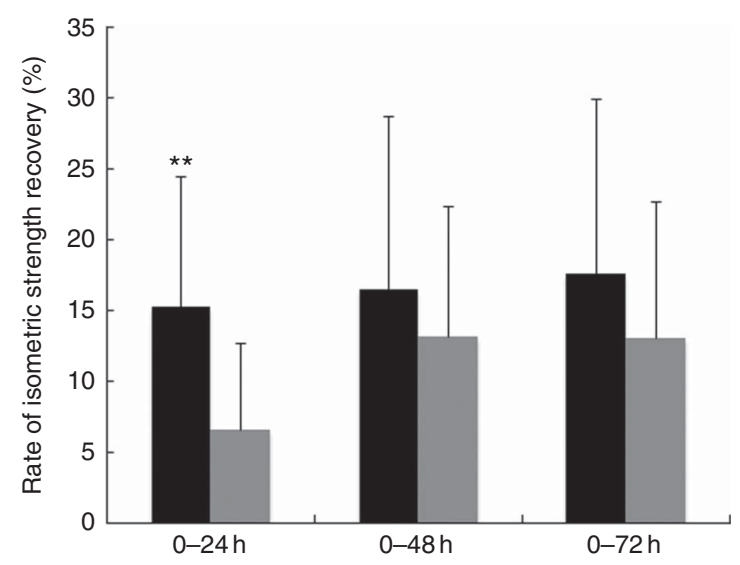

Fig. 3. Rate of strength recovery of isometric elbow flexion in the mate tea (MT, and control (CON, ) trials. Treatments were consumed at a dosage of $200 \mathrm{ml}$, three times a day for $11 \mathrm{~d}$. Values are means and standard deviations in the $0-24,0-48$ and $0-72 \mathrm{~h}$ periods. Two-way repeated-measures ANOVA and Tukey's post hoc test. The mean value was significantly different from that the control trial: ${ }^{\star \star} P<0.01$.

CON, $P=0 \cdot 016)$. However, the levels of total phenolics were higher in MT than in CON at all time points (PRE: $d=1 \cdot 12$, $P=0.005 ; 24 \mathrm{~h}: d=0.59, P=0.014 ; 48 \mathrm{~h}: d=0.55, P=0.044 ;$ and $72 \mathrm{~h}: d=0 \cdot 80, P=0 \cdot 021)$.

The blood concentration of GSH did not change over time in the MT trial (Fig. 5). However, there was a significant treatment effect $(P=0.002)$ for GSH. The levels of GSH were higher in MT than in CON at PRE $(d=1.02, P=0.032)$ and at $48 \mathrm{~h}(d=0.86$, $P=0.012)$ and $72 \mathrm{~h}(d=1.08, P=0.002)$ after exercise. In the CON trial, GSH had a non-significant rise from PRE to $24 \mathrm{~h}$ after exercise and then decreased significantly at $48 \mathrm{~h}(P=0.040)$ and $72 \mathrm{~h}(P=0.009)$ after exercise.

There were no significant changes over time or differences between conditions in either GSSG levels or GSH:GSSG ratio in 


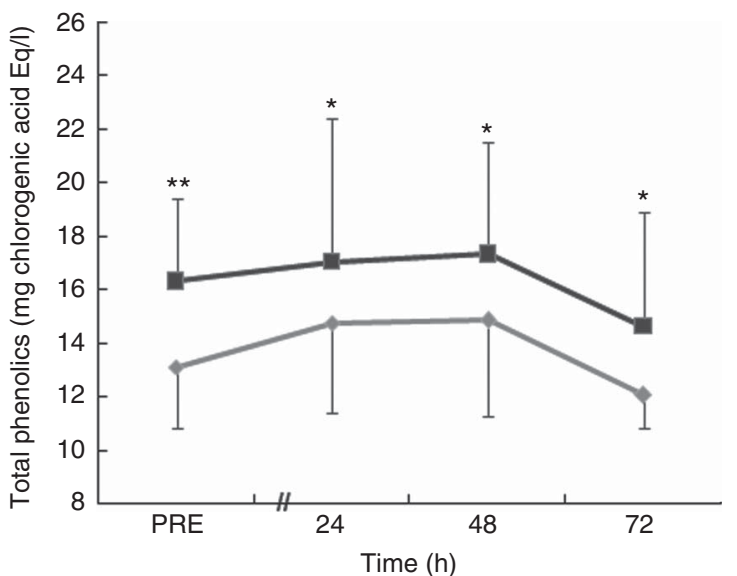

Fig. 4. Plasma concentration of total phenolics in the mate tea (MT, - and control $(\mathrm{CON},-)$ trials. Treatments were consumed at a dosage of $200 \mathrm{ml}$, three times a day for $11 \mathrm{~d}$. Values are means and standard deviations. Two-way repeated-measures ANOVA and Tukey's post hoc test. Significant time $(P<0.05)$ and treatment $(P<0.01)$ effects. The mean value was significantly different from that of the control trial: ${ }^{*} P<0.05$, ${ }^{\star *} P<0.01$.

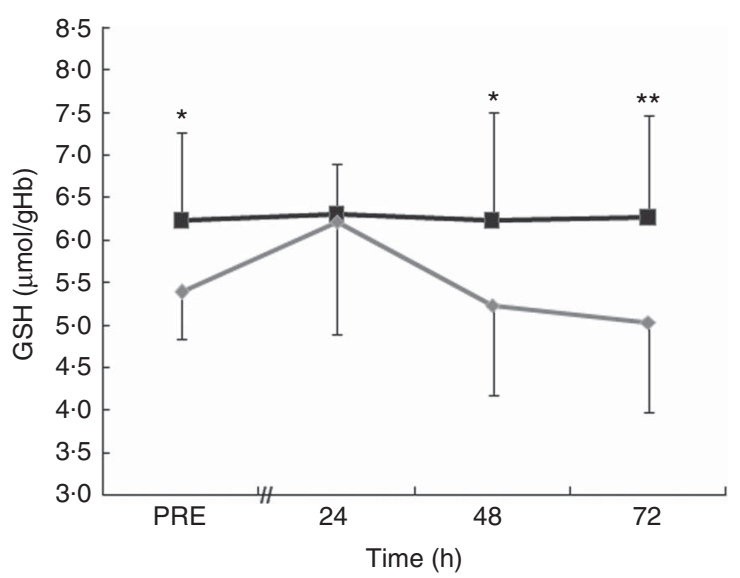

Fig. 5. Blood concentration of GSH in the mate tea (MT, - - - ) and control $(\mathrm{CON},--)$ trials. Treatments were consumed at a dosage of $200 \mathrm{ml}$, three times a day for $11 \mathrm{~d}$. Values are means and standard deviations. Two-way repeated-measures ANOVA and Tukey's post hoc test. Significant treatment $(P<0.01)$ effect. The mean value was significantly different from that of the control trial: ${ }^{\star} P<0.05,{ }^{\star \star} P<0.01$. the blood (Table 3 ). However, there was a medium effect size for GSSG at $72 \mathrm{~h}$ after exercise $(\delta=-0 \cdot 37)$. The effect size for GSSG was small at PRE $(\delta=-0 \cdot 25)$ and at $24 \mathrm{~h}$ after exercise $(\delta=-0.23)$ and negligible at $48 \mathrm{~h}(\delta=0.13)$ after exercise. In addition, effect size was medium for GSH:GSSG ratio at PRE $(\delta=0.38)$ and $72 \mathrm{~h}(\delta=0.42)$ but was small at $24 \mathrm{~h}(\delta=0 \cdot 28)$ and trivial at $48 \mathrm{~h}(\delta=0 \cdot 13)$ after exercise.

Plasma LOOH levels did not change over recovery time in either of the treatment trials (Fig. 6). LOOH levels were not significantly different between treatments at all time points after exercise. The effect sizes for LOOH were small at PRE $(d=-0 \cdot 20)$ and at $24 \mathrm{~h}(d=0 \cdot 21)$ and $48 \mathrm{~h}(d=-0 \cdot 45)$ after exercise and trivial $(d=0 \cdot 14)$ at $72 \mathrm{~h}$ after exercise.

\section{Discussion}

This study investigated the effect of MT intake, a beverage rich in antioxidant phytochemicals, on muscle strength recovery and oxidative stress markers after eccentric exercise in healthy subjects. Our primary finding was that the consumption of $600 \mathrm{ml}$ of MT (three $200 \mathrm{ml}$ drinks/d) providing $890 \mathrm{mg}$ of phenolic compounds/d improved the rate of recovery of isometric strength by $8.6 \%$ during the 1 st day after eccentric exercise. This positive effect of MT on muscle recovery was large and significant $(d=1.11 ; P=0.007)$ and is in agreement with reports that dietary phytochemical supplementation ameliorated muscle function after $\operatorname{EEIMD}^{(4,9-12)}$. In addition, although the measures of recovery were not significantly different between treatments (Fig. 2), MT had a medium effect $(d=0.53)$ on isometric strength at $24 \mathrm{~h}$ after exercise. This means that the influence of MT on muscle strength at $24 \mathrm{~h}$ after exercise was 'likely to be perceived' according to Cohen's statistics $^{(26)}$. The intake of MT also favoured the circulating levels of total phenols and GSH before and after eccentric exercise.

The mechanisms whereby MT influenced the recovery from EEIMD were not directly examined in the present study. However, antioxidant and/or anti-inflammatory properties of yerba mate might be involved. For example, MT intake has improved gene expression and activity of antioxidant enzymes in leucocytes ${ }^{(14,15)}$. MT has also reduced cytokine expression in macrophages $^{(31)}$ as well as inhibited neutrophil and macrophage infiltration into injured tissue ${ }^{(32)}$. After EEIDM,

Table 3. Oxidative stress markers' concentrations in blood at pre-exercise (PRE) and after eccentric* (Median values and interquartile ranges (IQR))

\begin{tabular}{|c|c|c|c|c|c|c|c|c|}
\hline & PRE & IQR (25-75\%) & $24 \mathrm{~h}$ & IQR (25-75\%) & $48 \mathrm{~h}$ & IQR (25-75\%) & $72 \mathrm{~h}$ & IQR (25-75\%) \\
\hline \multicolumn{9}{|c|}{ GSSG $(\mu \mathrm{mol} / \mathrm{g} \mathrm{Hb})$} \\
\hline MT & 0.01 & $0.01-0.02$ & 0.02 & $0.01-0.02$ & 0.02 & $0.01-0.03$ & 0.02 & $0.01-0.02$ \\
\hline CON & 0.02 & $0.01-0.02$ & 0.02 & $0.02-0.03$ & 0.02 & $0.01-0.02$ & 0.02 & $0.01-0.03$ \\
\hline$P$ & & 0.266 & & 0.240 & & 0.677 & & 0.110 \\
\hline \multicolumn{9}{|c|}{ GSH:GSSG ratio } \\
\hline MT & $526 \cdot 9$ & $340 \cdot 7-803 \cdot 3$ & 391.8 & $275 \cdot 1-772 \cdot 7$ & $282 \cdot 0$ & $197 \cdot 5-427 \cdot 6$ & $400 \cdot 0$ & $291 \cdot 8-718.9$ \\
\hline CON & 354.5 & $230 \cdot 1-568 \cdot 8$ & $303 \cdot 3$ & $268 \cdot 1-423 \cdot 5$ & $304 \cdot 1$ & $223 \cdot 3-603 \cdot 9$ & $312 \cdot 8$ & $128 \cdot 0-382 \cdot 7$ \\
\hline$P$ & & 0.176 & & 0.424 & & 0.831 & & 0.077 \\
\hline
\end{tabular}

MT, mate tea; CON, control.

* Friedman's and Wilcoxon's signed-rank tests. 


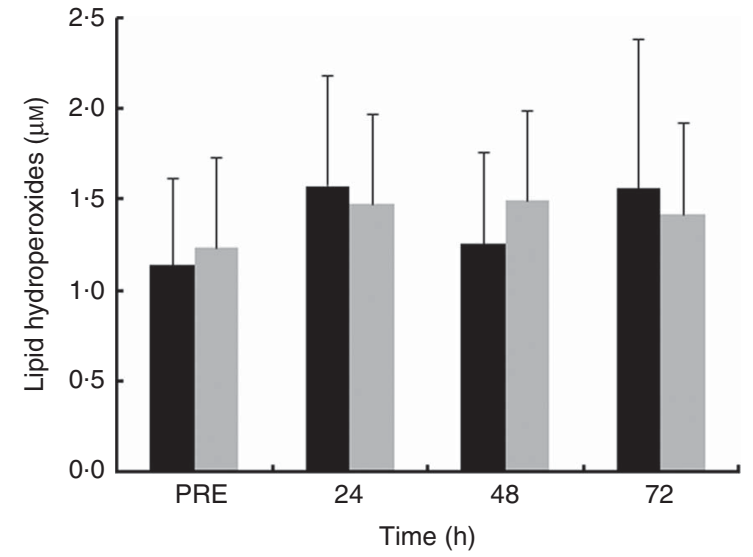

Fig. 6. Plasma concentration of lipid hydroperoxides in the mate tea (MT, and control $(C O N, \square)$ trials. Treatments were consumed at a dosage of $200 \mathrm{ml}$, three times a day for $11 \mathrm{~d}$. Values are means and standard deviations. Two-way repeated-measures ANOVA and Tukey's post hoc test.

phagocytes progressively accumulate in the muscle and initiate inflammatory events that precede tissue repair, including releasing reactive species that can help degrade cellular debris $^{(2,33-36)}$. It has been assumed that reactive species released by phagocytes could occasionally act on intact structures, leading to additional damage and delayed recovery of muscle strength ${ }^{(3,5-8,33)}$. Neutrophils are the first phagocytes to invade the muscle, typically peaking in concentration between 6 and $24 \mathrm{~h}$ after damage and then quickly decreasing in numbers ${ }^{(8,34-36)}$. Phagocytic macrophage concentrations are elevated at $24 \mathrm{~h}$ and peak about $48 \mathrm{~h}$ after injury ${ }^{(6,35,36)}$. In this study, the pattern of strength recovery during the first $24 \mathrm{~h}$ after exercise was improved in MT compared with CON (Fig. 2 and 3). Thus, one might speculate that the effect of MT on strength recovery was in part associated with the modulation of neutrophil activities in the muscles throughout the $24 \mathrm{~h}$ after EEIMD. The minor effectiveness of MT on recovery beyond $24 \mathrm{~h}$ after exercise would be in line with the decline in neutrophil numbers within the muscle after peaking in concentration ${ }^{(35)}$. However, significant amounts of neutrophils in damaged muscle have been found $5 \mathrm{~d}$ after eccentric exercise ${ }^{(34)}$. Thus, another explanation might be that, following the 1st day after exercise, the improvements on recovery promoted by MT would have been overwhelmed by an increment in reactive species generation from other sources (e.g. activated macrophages $)^{(35,36)}$ besides neutrophils.

The attenuation in MT effects on strength recovery beyond $24 \mathrm{~h}$ after exercise (Fig. 2) disagrees with the reports that supplementation with polyphenols-rich dietary sources improved muscle strength from 24 to $96 \mathrm{~h}$ after eccentric exercise $^{(4,9-12)}$. However, this limited influence of MT on recovery might be due to the extent of muscle damage ${ }^{(2)}$. After EEIMD, strength recovery can be halted for a few or several days depending on the injury severity ${ }^{(2,33)}$. In the present study, strength deficits were over $20 \%$ from 48 to $72 \mathrm{~h}$ after exercise in both treatments (Fig. 2). These results agree with the reports that supplementation with vegetable and anthocyanins-rich fruit powder concentrates for 4 weeks did not affect the losses of
$>30 \%$ in isometric strength over the $24-72 \mathrm{~h}$ after eccentric exercise $^{(37)}$. Conversely, the consumption of cherry juice - rich in anthocyanins - for $8 \mathrm{~d}^{(12)}$ or pomegranate juice - rich in ellagitannins - for $9 \mathrm{~d}^{(9)}$ improved isometric strength recovery through the 24-96-h period after eccentric exercise. In these studies $^{(9,12)}$, strength deficit at $72 \mathrm{~h}$ after exercise was about $16 \%$ with placebo, thus suggesting a lesser extent of muscle damage $^{(2)}$ compared with those seen in the present study as well as in the study by Goldfarb et al. ${ }^{(37)}$. It is noteworthy that the degree of EEIMD depends on several factors including the exercise protocol used ${ }^{(2)}$, which could in part explain the discrepancies between the studies regarding the degree of damage, and thus treatment effectiveness.

Significant changes in blood GSH, GSSG and/or GSSG:GSH ratio over $4 \mathrm{~d}$ after eccentric exercise have been reported by several studies ${ }^{(38,39)}$, whereas others have found just a transitory ${ }^{(37)}$ or no change in blood glutathione status ${ }^{(40)}$. In the present study, glutathione status seems to have been influenced by both the treatment and the exercise (Fig. 5). MT prevented the decrease in blood GSH from 24 to $72 \mathrm{~h}$ after exercise. In addition, MT had a significant influence on GSH before and at 48 and $72 \mathrm{~h}$ after exercise, as indicated by the large effect sizes ( $d=1.02,0.86$ and 1.08 , respectively). Conversely, neither GSSG nor GSH:GSSG ratio was affected in both trials (Table 3), which could be partially explained by the large variability (approximately 50-80\%) found in both blood markers at all time points. However, there was a medium effect size for GSSG levels at $72 \mathrm{~h}(\delta=0.32)$ that was expressed as a reduction of $21 \%$ in the median of GSSG with MT. In addition, the medium effect sizes for GSH:GSSG ratio before $(\delta=0.38)$ and at $72 \mathrm{~h}$ $(\delta=0 \cdot 42)$ after exercise corresponded to 50 and $90 \%$ increases, respectively, in the median of GSH:GSSG ratio in the MT trial. Overall, these results suggest that MT intake favoured blood glutathione status before and during the recovery period after EEIMD.

Among the phenolic compounds present in yerba mate infusions, there are many phenolic acids - mostly chlorogenic acids - and some flavonoids such as rutin, quercertin and kaempferol $^{(41,42)}$. These phytochemicals are highly effective in inhibiting the lipoperoxidation chain ${ }^{(43)}$. The large $(d=1.02)$ and significant effect of MT on the rest levels of plasma total phenolics after an 11-d treatment period (Fig. 4) agrees with the benefits provided by yerba mate in previous short-term studies $^{(14,15)}$. In addition, total phenolics were significantly higher with MT at all time points after exercise, showing medium effects at $24 \mathrm{~h}(d=0.59)$ and $48 \mathrm{~h}(d=0.55)$ and a large effect $(d=0.80)$ at $72 \mathrm{~h}$ after exercise. Interestingly, in both treatments, total phenolics decreased significantly from 48 to $72 \mathrm{~h}$ after exercise, suggesting that phenolic compounds were useful during the recovery from EEIMD. It is noteworthy that, in the CON trial, the decline in plasma total phenolics from 48 to $72 \mathrm{~h}$ (Fig. 4) paralleled the decrease in blood GSH over the same time period (Fig. 5). Altogether, these results may suggest that the decrease in blood GSH availability led to a greater reliance on dietary antioxidants, notably lipid radical scavengers ${ }^{(43)}$. On the other hand, one could propose that MT phenolics had GSH-sparing actions, mainly after the 2nd day after exercise. 
Plasma LOOH levels did not change in either treatment at all time points (Fig. 6). In the study of Goldfarb et al. ${ }^{(37)}$, LOOH levels were unaffected over the $72 \mathrm{~h}$ after exercise, but plasma malondialdehyde (MDA) increased from 24 to $72 \mathrm{~h}$ after exercise in the placebo trial. Conversely, Childs et al. ${ }^{(44)}$ found significant rise in $\mathrm{LOOH}$ but not in MDA in plasma from 48 to $96 \mathrm{~h}$ after eccentric exercise. These discrepancies concerning blood oxidative stress markers after eccentric exercise have been imputed to a number of factors such as exercise protocol, time point in blood sampling and the oxidative stress marker examined ${ }^{(38)}$.

The effects of MT on the biomarkers assessed in this study may have been limited by the small sample size. The lack of a placebo treatment may also have influenced the results, but unfortunately no suitable inert material compatible with the colour and flavour of the lyophilised MT was available. Nevertheless, in addition to have favoured blood antioxidant status, the bioactive properties of yerba mate may also have played some relevant role in the muscle after EEIMD, as suggested by the faster rate of strength recovery during the 1st day after eccentric exercise with mate intake (Fig. 1 and 2).

In conclusion, the consumption of MT increased blood antioxidant levels and improved the rate of strength recovery from 0 to $24 \mathrm{~h}$ after exercise. However, MT intake did not affect the strength recovery measures made from 24 to $72 \mathrm{~h}$ after eccentric exercise. Having a faster recovery rate of muscle function over the 1st day after eccentric exercise might be particularly important for subjects who need to perform subsequent exercises and/or occupational activities that will involve the previously damaged muscles. Thus, MT intake may be an interesting option for physically active subjects. Further studies should corroborate our findings in larger samples as well as investigate cellular mechanisms underlying the potential effects of MT on muscle recovery from EEIMD.

\section{Acknowledgements}

The authors thank Leao Junior Co. for providing the lyophilised instant MT.

The authors declare that Leão Alimentos e Bebidas partially supported this study by providing the lyophilised instant MT.

V. P. P.: conception and design of the study; collection, analysis and interpretation of the data; F. D.: design of the exercise protocol, supervision of the exercise data collection and critical revision of the manuscript; A. C. T.: design of the exercise protocol and collection of exercise data; C. d. Q. C.: biochemical analysis; H. S. B.: biochemical analysis; B. M. d. M.: collection of exercise data; R. L. S.: collection of exercise data; M. V. d. O.: biochemical analysis; E. d. O. P.: biochemical analysis; E. A. N.: supervision of the biochemical analysis and critical revision of the manuscript; E. L. d. S.: supervision of the biochemical analysis, interpretation of data, critical revision of the manuscript and approval of the final version of the manuscript.

None of the authors has any conflict of interest to declare.

\section{References}

1. Allen DG (2001) Eccentric muscle damage: mechanisms of early reduction of force. Acta Physiol Scand 171, 311-319.
2 Paulsen G, Mikkelsen UR, Raastad T, et al. (2012) Leucocytes, cytokines and satellite cells: what role do they play in muscle damage and regeneration following eccentric exercise? Exerc Immunol Rev 18, 42-97.

3. Faulkner JA, Brooks SV \& Opiteck JA (1993) Injury to skeletal muscle fibers during contractions: conditions of occurrence and prevention. Phys Ther 73, 911-921.

4. McLeay Y, Barnes MJ, Mundel T, et al. (2012) Effect of New Zealand blueberry consumption on recovery from eccentric exercise-induced muscle damage. J Int Soc Sports Nutr 9, 19.

5. Zerba E, Komorowski TE \& Faulkner JA (1990) Free radical injury to skeletal muscles of young, adult, and old mice. Am J Physiol 258, 429-435.

6. Lapointe BM, Frenette J \& Côté CH (2002) Lengthening contraction-induced inflammation is linked to secondary damage but devoid of neutrophil invasion. J Appl Physiol 92, 1995-2004.

7. Toumi H, F'guyer S \& Best TM (2006) The role of neutrophils in injury and repair following muscle stretch. J Anat 208, 459-470.

8. Pizza FX, Peterson JM, Baas JH, et al. (2005) Neutrophils contribute to muscle injury and impair its resolution after lengthening contractions in mice. J Physiol 562, 899-913.

9. Trombold JR, Barnes JN, Critchley L, et al. (2010) Ellagitannin consumption improves strength recovery $2-3 \mathrm{~d}$ after eccentric exercise. Med Sci Sports Exerc 42, 493-498.

10. Machin DR, Christmas KM, Chou TH, et al. (2014) Effects of differing dosages of pomegranate juice supplementation after eccentric exercise. Physiol J 2014, 7.

11. Trombold JR, Reinfeld AS, Casler JR, et al. (2011) The effect of pomegranate juice supplementation on strength and soreness after eccentric exercise. J Strength Cond Res 25, 1782-1788.

12. Connolly DA, McHugh MP, Padilla-Zakour OI, et al. (2006) Efficacy of a tart cherry juice blend in preventing the symptoms of muscle damage. Br J Sports Med 40, 679-683.

13. Bracesco N, Sanchez AG, Contreras V, et al. (2011) Recent advances on Ilex paraguariensis research: minireview. J Ethnopharmacol 136, 378-384.

14. Matsumoto RL, Bastos DH, Mendonça S, et al. (2009) Effects of mate tea (Ilex paraguariensis) ingestion on mRNA expression of antioxidant enzymes, lipid peroxidation, and total antioxidant status in healthy young women. J Agric Food Chem 57, 1775-1780.

15. Fernandes ES, Machado MO, Becker AM, et al. (2012) Yerba mate (Ilex paraguariensis) enhances the gene modulation and activity of paraoxonase-2: in vitro and in vivo studies. Nutrition 28, 1157-1164.

16. Klein GA, Stefanuto A, Boaventura BC, et al. (2011) Mate tea (Ilex paraguariensis) improves glycemic and lipid profiles of type 2 diabetes and pre-diabetes individuals: a pilot study. J Am Coll Nutr 30, 320-332.

17. Alkhatib A (2014) Yerba Maté (Illex paraguariensis) ingestion augments fat oxidation and energy expenditure during exercise at various submaximal intensities. Nutr Metab (Lond) 11, 42 .

18. Morgan-Martins MA, Silva VS, Licks S, et al. (2013) Ilex paraguariensis St. Hill (yerba mate) improves the metabolic profile of and protects against oxidative stress in exercised and non-exercised rats. Int J Pharm Bio Sci 4, 1303-1311.

19. Singleton VL, Orthofer R \& Lamuela-Raventos RM (1999) Analysis of total phenols and other oxidation substrates and antioxidants by means of Folin-Ciocalteu reagent. Methods Enzymol 299, 152-178.

20. Strassmann BB, Vieira AR, Pedrotti EL, et al. (2008) Quantitation of methylxanthinic alkaloids and phenolic compounds in mate (Ilex paraguariensis) and their effects on 
blood vessel formation in chick embryos. J Agric Food Chem 56, 8348-8353.

21. Gnoatto SCB, Schenkel EP \& Bassani VL (2005) HPLC method to assay total saponins in Ilex paraguariensis aqueous extract. J Braz Chem Soc 16, 723-726.

22. Fan JP \& He CH (2006) Simultaneous quantification of three major bioactive triterpene acids in the leaves of Diospyros kaki by high performance liquid chromatography method. J Pharm Biomed Anal 41, 950-956.

23. Serafini M, Maiani G \& Ferro-Luzzi A (1998) Alcohol-free red wine enhances plasma antioxidant capacity in humans. $J$ Nutr 128, 1003-1007.

24. Giustarini D, Dalle-Donne I, Milzani A, et al. (2013) Analysis of GSH and GSSG after derivatization with N-ethylmaleimide. Nat Protoc 8, 1660-1669.

25. Nourooz-Zadeh J, Tajaddini-Sarmadi J \& Wolff SP (1994) Measurement of plasma hydroperoxide concentrations by the ferrous oxidation xylenol orange assay in conjunction with triphenylphosphine. Anal Biochem 220, 403-409.

26. Cohen J (1993) A power primer. Psychol Bull 112, 155-159.

27. Dunlap WP, Cortina JM, Vaslow JB, et al. (1996) Meta-analysis of experiments with matched groups or repeated measures designs. Psychol Methods 1, 170-177.

28. Romano J, Kromrey JD, Coraggio J, et al. (2006) Exploring methods for evaluating group differences on the NSSE and other surveys: are the t-test and Cohen's d indices the most appropriate choices? http://www.coedu.usf.edu/main/ departments/me/documents/methodsforevaluatinggroup.pdf (accessed December 2014).

29. Faul F, Erdfelder E, Lang A-G \& Buchner A (2007)) G*Power 3: a flexible statistical power analysis program for the social, behavioral, and biomedical sciences. Behav Res Methods 39, 175-191.

30. MacBeth G, Razumiejczyk E \& Ldesma RD (2011) Cliff 's Delta Calculator: a non-parametric effect size program for two groups of observations. Univ Psychol 10, 545-555.

31. Borges MC, Vinolo MA, Nakajima K, et al. (2013) The effect of mate tea (Ilex paraguariensis) on metabolic and inflammatory parameters in high-fat diet-fed Wistar rats. Int J Food Sci Nutr 64, 561-569.

32. Lanzetti M, Bezerra FS, Romana-Souza B, et al. (2008) Mate tea reduced acute lung inflammation in mice exposed to cigarette smoke. Nutrition 24, 375-381.
33. MacIntyre DL, Reid WD, Lyster DM, et al. (1996) Presence of WBC, decreased strength, and delayed soreness in muscle after eccentric exercise. J Appl Physiol 80, 1006-1013.

34. Fielding RA, Manfredi TJ, Ding W, et al. (1993) Acute phase response in exercise. III. Neutrophil and IL-1 beta accumulation in skeletal muscle. Am J Physiol 265, 166-172.

35. Tidball JG \& Villalta SA (2010) Regulatory interactions between muscle and the immune system during muscle regeneration. Am J Physiol Regul Integr Comp Physiol 298, 1173-1187.

36. Brickson S, Hollander J, Corr DT, et al. (2001) Oxidant production and immune response after stretch injury in skeletal muscle. Med Sci Sports Exerc 33, 2010-2015.

37. Goldfarb AH, Garten RS, Cho C, et al. (2011) Effects of a fruit/ berry/vegetable supplement on muscle function and oxidative stress. Med Sci Sports Exerc 43, 501-508.

38. Paschalis V, Nikolaidis MG, Fatouros IG, et al. (2007) Uniform and prolonged changes in blood oxidative stress after muscle-damaging exercise. In Vivo 21, 877-883.

39. Nikolaidis MG, Paschalis V, Giakas G, et al. (2007) Decreased blood oxidative stress after repeated muscle-damaging exercise. Med Sci Sports Exerc 39, 1080-1089.

40. Lee J, Goldfarb AH, Rescino MH, et al. (2002) Eccentric exercise effect on blood oxidative-stress markers and delayed onset of muscle soreness. Med Sci Sports Exerc 34, 443-448.

41. Bastos DH, Saldanha LA, Catharino RR, et al. (2007) Phenolic antioxidants identified by ESI-MS from Yerba maté (Ilex paraguariensis) and green tea (Camellia sinensis) extracts. Molecules 12, 423-432.

42. Filip R, López P, Giberti G, et al. (2001) Phenolic compounds in seven South American Ilex species. Fitoterapia 72, 774-778.

43. Rice-Evans CA, Miller NJ \& Paganga G (1996) Structureantioxidant activity relationships of flavonoids and phenolic acids. Free Radic Biol Med 20, 933-956.

44. Childs A, Jacobs C, Kaminski T, et al. (2001) Supplementation with vitamin $\mathrm{C}$ and $\mathrm{N}$-acetyl-cysteine increases oxidative stress in humans after an acute muscle injury induced by eccentric exercise. Free Radic Biol Med 31, 745-753. 